\section{Dishing a modern myth about microbes}

SIR - We are indebted to Lynn J. Rothschild for debunking, in Journal Club, some microbial myths (Nature 443, 249; 2006). Her prime concern is that many microbiologists have simply given up trying to culture many of the more recalcitrant microbes living in the natural environment, in favour of resorting to DNA-sequencing methods.

There is a lingering and widespread presumption that DNA-sequence information will make a huge difference to our knowledge of microbes in the natural world, but we, like Rothschild, beg to differ. She argues for a greater effort to learn how to culture the 'unculturable' microbes. We would go further, and suggest that, having done so, we should devote greater effort to describing species by their phenotypic properties. Characterization of the phenotype, rather than the genetic sequence, is the key to gaining deeper and better understanding of microbial diversity in the natural world. Tom Fenchel ${ }^{\star}$, Genoveva F. Estebant', Bland J. Finlay

* Marine Biological Laboratory, University of Copenhagen, DK-3000 Helsingør, Denmark †CEH-Dorset, Winfrith Technology Centre, Dorchester, Dorset DT2 8ZD, UK

\section{The daunting process of MIAME}

SIR - Michael Eisenstein, in his Technology Feature "Quality control" (Nature 442, 10671070; 2006), reports recent improvements in microarray technologies. He discusses the establishment of systems for "clearly defining how [microarray] data were obtained", such as the MIAME (minimum information about a microarray experiment) annotation standards.

There are two problems inherent to the use of such standards. First, microarrays generate datasets whose intrinsic dimensionalities are several orders of magnitude greater than the information content of the standards. Second, one cannot know a priori all the biological factors that might affect the results reported by microarrays.

In terms of the first point, the prospect of defining 30,000-odd different MIAME descriptors - the routine size of microarrays - would be daunting under any circumstances. The second point is neatly illustrated by work described in the same issue (J. Molinier, G. Ries, C. Zipfel and B. Hohn Nature 442,1046-1049; 2006). This study reported the unexpected finding of transgenerational, non-genetic memory of stress events in the flowering plant Arabidopsis thaliana. Although transcriptome analyses in this case did not detect differences in global gene expression (possibly due to lack of sensitivity), the question remains as to whether one should be required to record the prior generational treatments of plant lines in microarray experiments - or in any experiment. Such a requirement appears equally daunting.

How should we proceed? Reducing the costs of microarray technology so that experiments can be readily reproduced across laboratories seems a reasonable approach. Relying on minimal standards of annotation such as MIAME seems unreasonable, and should be abandoned.

David W. Galbraith

Bio5 Institute and Department of Plant Sciences, University of Arizona, Tucson, Arizona 85721, USA

\section{No room for complacency on drug resistance in Africa}

SIR - Your News Feature "Staying the course" (Nature 442, 617-619; 2006) offered a welcome dose of optimism in the debate about mass deployment of antiretroviral therapies in Africa, by arguing that this had not yet created an epidemic of drug-resistant HIV. But although everyone in the field has been delighted by initial studies suggesting high adherence, we must not be complacent.

The News Feature cited studies showing that $70-80 \%$ of patients on antiretroviral drugs had undetectable viral loads; the converse is that $20-30 \%$ have detectable viral loads. The spread of resistance is determined not only by compliance - which, as you point out, has been good so far in most settings - but also by factors more specific to Africa, such as interactions between HIV and TB drugs, or by limitations of drug-supply infrastructure. The predominant use of clinical monitoring in African programmes may allow mutations to accumulate (DART Virology Group AIDS 20, 1391-1399; 2006), potentially making resistant strains more transmissible. If virological failure accompanies the roll-out of these drugs, it could rapidly increase with mass deployment: see how fast this might happen at http://pcwww.liv.ac.uk/ hastings/HIV_models/2006_HIVdynamics. xls. Scientists must pool data from roll-out cohorts (functioning as sentinel sites), not only to assess resistance but also to gather data on adherence, pharmacokinetics, drug interactions and other factors that determine a drug regimen's useful lifespan.

The consequences of widespread use of nevirapine to prevent mother-to-child HIV transmission are not yet clear, and a public health fall-back plan is urgently needed in case large-scale resistance emerges. Wellfunded education programmes are vital to help people minimize their risk of infection, encourage them to undergo the counselling and testing they need to gain access to antiretrovirals, and overcome the stigma of AIDS. The success of initial antiretroviral deployment is encouraging, but we should not underestimate the challenges that remain. Ian M. Hastings ${ }^{\star}$, David G. Lalloo*,

Saye H. Khoo'

*Liverpool School of Tropical Medicine,

Pembroke Place, Liverpool L3 5QA, UK

$\uparrow$ Department of Pharmacology and Therapeutics, University of Liverpool, Liverpool L69 3BX, UK

\section{Brief: goodbye to a quirky perspective on science}

SIR - I am writing to protest at your decision to cancel the Brief Communications section (Nature 443, 246; 2006). I have always enjoyed the high-quality yet rather quirky things published there. Where else would I learn about synchronized hand-clapping, the photonic band structure of beetle exoskeletons or mathematical models of bilingual societies? I turn to the Brief Communications section to see ways in which rigorous scientific approaches can shed light on very novel questions.

I have not yet come across something appropriate in my own research to submit as a Brief Communication, so I suppose that, having done nothing to alleviate the quality problem, I have no right to complain. (I am working in my spare time on a result that fits the spirit of the section, being a mathematical proof that there's a 'best' way to vote, but I can't think of a way to reduce it to a single page.) Still, I doubt I'm the only reader who's disappointed by this loss.

Brief Communications have always served to remind me that science can be both excellent and quirky. When I was teaching optics and discussing novel optical phenomena in the animal kingdom, I would show students the article "Opal analogue discovered in a weevil" (Nature 426, 786-787; 2003). The second page included a Brief Communication titled "Health benefits of eating chocolate?". Without Brief Communications, how would I be able to teach students both that insects are talented optical engineers and that chocolate may be good for your health?

Alex Small

Laboratory of Integrative and Medical Biophysics, National Institute of Child Health and Human Development, Bethesda, Maryland 20892, USA

\section{Brief Communications (RIP) and the soul of wit}

\author{
SIR — "What a pity," he said — briefly. \\ Jeremy Wolfe \\ Visual Attention Lab, Brigham \& Women's \\ Hospital, Cambridge, Massachusetts 02139, USA
}

